\title{
Numerical differentiation of experimental data: local versus global methods
}

\author{
Karsten Ahnert, Markus Abel \\ Institute of Physics, University of Potsdam, 14415 Potsdam, Germany
}

\begin{abstract}
In the context of the analysis of measured data, one is often faced with the task to differentiate data numerically. Typically, this occurs when measured data are concerned or data are evaluated numerically during the evolution of partial or ordinary differential equations. Usually, one does not take care for accuracy of the resulting estimates of derivatives because modern computers are assumed to be accurate to many digits. But measurements yield intrinsic errors, which are often much less accurate than the limit of the machine used, and there exists the effect of "loss of significance", well known in numerical mathematics and computational physics. The problem occurs primarily in numerical subtraction, and clearly, the estimation of derivatives involves the approximation of differences. In this article, we discuss several techniques for the estimation of derivatives. As a novel aspect, we divide into local and global methods, and explain the respective shortcomings. We have developed a general scheme for global methods, and illustrate our ideas by spline smoothing and spectral smoothing. The results from these less known techniques are confronted with the ones from local methods. As typical for the latter, we chose Savitzky-Golay filtering and finite differences. Two basic quantities are used for characterization of results: The variance of the difference of the true derivative and its estimate, and as important new characteristic, the smoothness of the estimate. We apply the different techniques to numerically produced data and demonstrate the application to data from an aeroacoustic experiment. As a result, we find that global methods are generally preferable if a smooth process is considered. For rough estimates local methods work acceptably well.
\end{abstract}

Key words: numerical differentiation, data analysis, filtering methods, smoothing splines, nonparametric regression

PACS: 02.60.Ed, 02.60.Jh, 29.85.+c, 07.05.Kf

Email addresses: kahnert@stat.physik.uni-potsdam.de (Karsten Ahnert), markus@stat . physik. uni-potsdam.de (Markus Abel).

Preprint submitted to Computer Physics Communications 2 February 2008 


\section{Introduction}

The estimation of derivatives from numerical data is a classical problem which occurs in many problems of data analysis [1]. Applications range from biology [2,3], and chemistry [4] to a variety of problems in physical applications $[5,6,7]$; various mathematical aspects are discussed in $[8,9,10,11]$. Surprisingly, experimenters and numerical data analysts often use very crude techniques for the estimation of derivatives and the topic is not discussed in depth in the physics community. Despite the relatively simple strategies to enhance results for numerical differentiation, improved techniques are rarely used. With this contribution, we formulate a general scheme to distinguish the existing methods and discuss in detail their differences. Our emphasis is on accuracy and smoothness, so wherever one is not bothered with very accurate estimation or with very noisy data, our text is understood to be informative. However, in critical applications with a high standard on accuracy of the data, this paper can guide along the numerical subtleties of the estimation of derivatives with a minimum loss of accuracy. The task we try to analyze is to differentiate numerically a data series with given accuracy in such a way that the loss of accuracy is minimal and the smoothness of the result, defined later in this text, is maximal. In the following, we present typical techniques and algorithms in a computation-oriented way, referring to the literature for mathematical details.

Whereas mathematically, the derivative of a function is obtained by a limit process involving infinitesimal calculus, this can never be realized for data measured by digital equipment due to the intrinsic discretization of the data. In addition, real measurements yield data with noise, either due to the device properties or due to finite resolution, e.g., when sampling the data. An estimate for a derivative of some data $y(x)$ with respect to the argument $x$ has to cope with these two restrictions. Noise and discretization effects yield errors in the data $y$, the finite sampling interval does not allow the limit $\Delta x \rightarrow 0$ and thus produces numerical errors, too.

Typically, to obtain an estimate for the derivative $y^{\prime}(x)$, one tries to approximate the measured data $y$ best (in an exactly specified sense). One then hopes that the derivative found from this approximation is as well best. In this article, we want to obtain maximal accuracy, but as well the smoothest result possible. One can divide the existing techniques into local and global strategies. For the first, one fits or interpolates a function through the data points locally, as for a running window; in the second case, the fit or interpolation is global. A typical example for global fits are smoothing splines as an enhancement of the well-known interpolating splines [12]. In contrast to interpolation a spline is put through many data fulfilling a least-squares condition. This way, one can take into account a natural property of physical variables - their smoothness, which is very often assumed for theoretical or experimental rea- 
sons. Taken into account this criterion, differences between methods can be clearly noticed. The quantification of the smoothness property is novel to the numerical differentiation, we believe that it is an important and useful criterion. Another quantification of the quality of the estimate than smoothness is the least-squares error. A consistent formulation including a smoothness parameter yields a minimization problem, mathematically known as Tikhonov regularization $[11,13]$.

Sometimes, a kernel smoother is applied before fitting the data. This is a quite crude technique and we can not recommend this method, since two regression methods are combined and one carries the disadvantages of both methods. Furthermore, most methods work better with the original data [14]. But we must also note, that some combinations of kernel smoothers and regression methods might be successfully applied to some special problems.

In this article, we compare four different techniques: finite differencing [15], the Savitzky-Golay filter [14], smoothing splines [12] and a spectral technique $[5,14]$. Whereas the first two are local methods, the latter are global ones. Local methods typically produce non smooth functions, whereas for global methods the smoothness is controlled in a well-defined way. We compare the methods by two numerically produced data sets where the noise in the data can be controlled and the derivative is known either analytically or numerically with high accuracy. Finally, we apply the methods to a set of data from an aeroacoustic measurement [16].

This article is organized as follows: In Section 2, we give a brief overview of the topic and details on local and global methods. Results for the implementation of the methods are given in Section 3 for the three above mentioned examples. The article concludes with Section 4.

\section{Estimation of Derivatives}

The estimation of derivatives is frequently encountered in the numerical integration of ordinary differential equations [14, Chp. 5,16]. A function $f(x)$ is given and one has to compute its derivative. For this task, the typical estimators like finite difference schemes, spectral or kernel methods are used $[15,14,17]$ in dependence on the required accuracy and speed of the numerics. The key problem concerning accuracy is the cancellation of digits with numerical subtraction, mentioned above. An introductory analysis is given in [14, Chp. 5], a more detailed exposure is found in $[14,18,19]$.

A conceptually different problem arises if one wants to estimate derivatives from measured data without knowing the underlying function. Then, one needs 
first a "good" estimate for $f(x)$ and then another "good" method to extract the derivative. What "good" means can vary heavily from data set to data set. The algorithms and methods one uses to solve this problem depend on the requirements the analyst imposes on the data. E.g., it might be unimportant for an experimenter if his result is accurate up to 2 or 10 digits because he only needs a graphical representation of the results. On the other hand, some applications, like differential embedding [20], might require the knowledge of several derivatives at high accuracy for further analysis of a dynamical system. Then, usual techniques, as given in [14] very quickly meet their limits regarding accuracy and smoothness of a numerically calculated derivative.

In the mathematical literature, the problem has been analyzed as inverse problem under numerical aspects $[11,14,21]$. Here, we follow the rigorous ideas of [11] and present a classification of the schemes into global and local ones; we illustrate the differences by typical examples. Our focus is on the smoothness of the estimated derivatives, because a description of a physical system typically requires functions and derivatives to be smooth up to a certain order. For example, most Hamiltonian systems are smooth systems, since their solutions are mostly required to be $C^{2}$.

In the following, we consider a data series $y(x)$, measured at $N$ points $\left(y_{i}, x_{i}\right)$ $(i=1, \ldots, N)$. Due to measurement accuracy and noise sources the data have errors and we assume that our measured values consist of a deterministic part $f(x)$ and a noisy part $\eta(x)$, such that the measured data are $y(x)=f(x)+$ $\eta(x)$. This already indicates that the true function $f(x)$ should be found using regression techniques. Since $f$ is generically nonlinear, linear regression [14] is not applicable and nonparametric methods should be used; for an introduction see [22]. Please note that this is a much more complex problem than the estimation of parameters $\alpha_{i}, i=1, \ldots, n$ by iterative methods if the form of $f\left(x, \alpha_{1}, \ldots, \alpha_{n}\right)$ is known, as, e.g., the standard Levenberg-Marquardt one $[14,23,24]$. We assume further that $f$ is a smooth function and that the data series $y_{i}\left(x_{i}\right)$ is given on a uniform grid $x_{i}=a+i \Delta x, i=0,1,2, \ldots$ of length $L=b-a$ in the interval $a \leq x_{i} \leq b$. Now, we are interested in the estimate for the derivatives $f^{\prime}(x)=\mathrm{d} f / \mathrm{d} x$ from the measured data. Throughout this article we will denote the estimate of the derivative by $\tilde{f}^{\prime}(x)$ and accordingly the estimate of the function by $\tilde{f}(x)$.

After having experimented with many data sets and methods, we found out that the techniques commonly used can be distinguished in a simple way as local or global approximation methods. A local method approximates the function $f(x)$ in some neighborhood $\mathcal{U}(x)$ of $x$, and does not yield information beyond. This means especially, that there can be jumps in the approximated function between different $x$ values. A global method includes information about all points in the estimate for $f(x)$ and thus jumps can be avoided if smooth functions are used for the approximation. 
The basic idea in all the methods is to approximate the function $f(x)$ with the hope that then the derivative can be estimated. More explicitly one assumes the following: if the approximation $\tilde{f}$ is close to the original function $f$, then the derivative $\tilde{f}^{\prime}$ is also close to $f^{\prime}$. In mathematical terms, if $\|f-\tilde{f}\|=\min$, then $\left\|f^{\prime}-\tilde{f}^{\prime}\right\| \approx \min$, with $\|\cdot\|$ a suitable norm. The estimation is best in the sense of the applied norm. Usually, the $L_{2}$ norm is chosen and one has to solve a least-squares problem $[14,23,24]$. Common methods yield functions $\tilde{f}$ obtained either by local or global interpolation, or a fit [14]. In an interpolation problem the resulting function is required to go through the data points, i.e. $\tilde{f}\left(x_{i}\right)=y_{i}$; a fit produces the function such that $\sum_{j}\left\|\tilde{f}\left(x_{j}\right)-y_{j}\right\|=\min$. Implicitly, that means that $\tilde{f}\left(x_{i}\right)$ is not necessarily identical to $y_{i}$. A local method acts on a subset of the data $\left(j \in\left(i-n_{l}, i+n_{r}\right)\right)$, with $n_{r}, n_{l} \in \mathbb{N}$ a global one on all available data. Most of the methods can be extended to give approximate function values or derivatives not only at the points $x_{i}$ but on the whole interval $a \leq x \leq b$. At the boundaries some methods are problematic because, e.g., the statistic changes, or the problem is no longer well posed.

\subsection{Local Methods}

Local methods work by fitting or interpolating for each $x_{i}$ a function $\tilde{f}(x)$ on some sub-interval $I \ni x$ of the domain. Obviously, this does not guarantee that the function is smooth, because the measurement errors can yield jumps (from interval to interval). Probably the best known method is given by finite differences; it results in a natural way when differential operators are discretized. This technique has turned to a huge field in connection with numerical integration of partial differential equations [15]. Depending on the problem, different schemes can be used and the choice of the right difference scheme can have enormous impact on the result of an integration [25]. The basic idea is simple: the function $f$ is interpolated by a polynomial of order $m$. For symmetric differencing, it is put through the points $x_{i-k}, \ldots, x_{i}, \ldots, x_{i+k}$, where $m=2 k$ is a positive integer, asymmetric schemes, like the well-known upwind schemes work in a similar way. The derivative at the point $x$ is then the derivative of the interpolated polynomial. Finite differencing is local, because the approximation of the derivative depends only on the $2 k+1$ points in the neighborhood of $x_{i}$. The polynomial can be obtained from Taylor expansion, Padé approximation or similar schemes [25]. The finite difference estimator for a Taylor expansion has the form [15]

$$
\left(\frac{\partial \tilde{f}}{\partial x}\right)_{i}=\sum_{j=1}^{k} \alpha_{j} \frac{y_{i+j}-y_{i-j}}{2 j \Delta x}
$$


with coefficients

$$
\alpha_{j}=2 \cdot(-1)^{j+1}\left(\begin{array}{c}
k \\
k-j
\end{array}\right) /\left(\begin{array}{c}
k+j \\
k
\end{array}\right) .
$$

For other schemes, different coefficients are used. In that case (2) or the right hand side of (1) can contain derivatives. It is also possible to vary the step width $\Delta x$. To do so, one replaces $\Delta x$ by $\delta x=l \Delta x(l \in \mathbb{N})$ and $j$ by $j^{\prime}=l j$ in (1).

If the data are noise-free, the error $e=\left\|f^{\prime}-\tilde{f}^{\prime}\right\|$ in (1) is of order $O\left(\Delta x^{m}\right)$. This means, with a fine sampling one can arrive at accurate approximations. Numerically, however, one is faced with the unavoidable problem of accuracy loss in numeric subtraction due to the loss of significance of digits $[18,19]$. Subtraction of two floating point numbers is ill conditioned if the value of two numbers is approximately equal. This is typical for the scheme (1). Especially if measurement noise is present, it can easily travel to the leading digits and render the results meaningless; i.e. finite differencing is a bad choice for numerical differentiation. One can consider the problem as a trade-off between numerical inaccuracy due to subtraction and analytical need for small values of $\Delta x$ for the approximation (1) to hold sufficiently well.

A consequent error analysis including the data accuracy $\delta=\sqrt{V A R(\eta)}$, with $\eta$ the measurement noise process, yields the error of a first-order symmetric finite difference scheme $(k=1)$ in Eq. (1) [11]:

$$
e \sim \delta x+\delta / \delta x
$$

A minimal error $e \simeq 2 \sqrt{\delta}$ is found for $\delta x \sim \sqrt{\delta}$. With given data of sampling interval $\Delta x \ll \sqrt{\delta}$ one needs to discard some points to achieve the minimum and uses $\delta x=l \Delta x$ This is not satisfying, because the information contained in the left-out points in the interval $y_{i-l}, \ldots, y_{i+l}$ is thrown away. One would like to use a scheme which has minimal error, but uses all points with the intention to go beyond the bound $2 \delta$ for the error of the estimate.

This can be achieved by using a fit of a polynomial of order $m<2 k$ through all data in the interval $\left(x_{i-k}, x_{i+k}\right)$. This method is known as Savitzky-Golayfiltering and is widely used in data analysis. The domain has not to be symmetric (as in generalized finite differencing), one defines a neighborhood by an interval $\left(i-n_{l}, i+n_{r}\right)$ with $n_{l}+n_{r}+1$ points, and $n_{l}$ not necessarily equal to $n_{r}$. A linear regression is used to find the best polynomial fit of order $m$ to the data, and the derivative is obtained from the coefficients of the polynomial. This procedure is repeated for every data point, like for a moving window. The key idea of Savitzky-Golay filtering is the conservation of higher statistical moments. A simple moving average always reduces the height of a local extremum. Due to the mentioned conservation property, the Savitzky-Golay 
filter shows this reduction to a much less extent. Smoothness, however, is not guaranteed and the derivative can be discontinuous, which is not desirable for an estimate useful in physical problems where $f$ is typically required to be smooth. A very nice and detailed discussion is found in the numerical recipes [14].

\subsection{Global Methods}

Global methods yield an estimation $\tilde{f}(x)$, defined on the whole interval $a \leq$ $x \leq b$. Since the function is not known beforehand, it makes sense to use a representation by some basis functions, $\phi_{i}(x), i \in \mathbb{N}: f(x)=\sum_{j} a_{j} \phi_{j}(x)$. The estimation shall be best in the least squares sense, but as well smooth. Consequently a minimization problem with a side condition for smoothness is formulated. The coefficients $a_{j}$ are determined accordingly. The choice of the basis depends on the properties on $f$, either known by prior knowledge oder imposed a posteriori. For instance, it might be clear from the experiment that $f \in C^{2}$ is smooth (in that the second derivative exists), or continuous only, or periodic on the interval, or has other restrictions which are known to the modeler.

To quantify the global smoothness of a function $g(x)$ one uses its curvature $[26]$

$$
s(g)=\int_{a}^{b}\left\|g^{\prime \prime}(x)\right\|^{2} \mathrm{~d} x .
$$

Maximizing the smoothness of an estimation amounts to minimizing its curvature. The function estimate $\tilde{f}(x)$ is then determined by the usual least-squares minimization problem with the additional smoothness constraint. The amount of smoothing is controlled by the smoothing parameter $\lambda$, which enters the minimization problem:

$$
\chi^{2}=\sum_{i}\left\{y_{i}-\tilde{f}\left(x_{i}\right)\right\}^{2}+\lambda \int_{a}^{b}\left\{\tilde{f}^{\prime \prime}(x)\right\}^{2} \mathrm{~d} x \stackrel{!}{=} \min .
$$

For simplicity, we have assumed equal measurement uncertainty for all data points $\sigma_{i}=$ const and neglected it in the formulas. The first term measures the least squares error of the fit, while the second term penalizes curvature in the function. Equation (5) is a a typical bias-variance problem [27], and the best choice of the smoothing parameter is nontrivial. Numerically, it can be determined using generalized cross-validation [28].

As mentioned above, $\tilde{f}$ is usually represented by a superposition of some basis functions with according coefficients, $a_{j}$. This is inserted into (5). The minimizing coefficients, $a_{j}$, are determined by a variational principle. As a consequence the conditions $\partial \chi^{2} / \partial a_{j}=0$ have to be fulfilled and the resulting 
set of equations needs to be solved (see App. A). If we require $f \in C^{2}$ to be twice differentiable, natural cubic splines are an obvious choice. For periodic functions, or $f \in C^{\infty}$, a spectral representation suits well. In other situations, other basis systems might be favorable. In the following we consider smoothing splines and Fourier representation.

We want to hint at this point that smoothing splines are different from the concept of interpolating splines. This is a very important point for the understanding of the following. Interpolating splines fit a polynomial through every data point - in our case a horrible scenario, since we are faced with noise. Smoothing splines fit a low-order polynomial through bunches of data, such that the resulting approximation is best in a least-square sense, cf. Eqs. (4) and (5). Summarizing, we note that the smoothing and the interpolating splines have the representation as low-order polynomial of nth order (typically 3rd order) in common. Differences lie in the construction. Whereas interpolation puts a smooth line between points, smoothing splines use regression to put the best "line" (or a n-dimensional surface, in general) through many points. Both, interpolating or smoothing ones, are global, because there is no jump in the nth-order spline over the whole definition range - nor in the n- 1 possible derivatives! Furthermore, the smoothing spline is constructed from a global measure (4). This measure couples the local spline coefficients.

In [11], it has been shown rigorously that the minimizer of (5) is a natural cubic spline, provided the function $f$ is square integrable. The spline representation used throughout this paper reads

$$
f(x)=\sum_{j=0}^{n+2} \gamma_{j} B_{j}(x),
$$

where $\gamma_{j}$ are the coefficients of the cubic B-spline basis functions $B_{j}(x)$ and $n$ is the number of knots for constructing the smoothing spline. After the solution of the minimization problem one calculates the derivative analytically from the basis functions. The property, important from a fundamental point of view is the smoothness of the splines. As shown in [11], $e \sim \sqrt{\delta}$ for $\Delta x \rightarrow 0$, which is superior to the accuracy (3) for finite difference methods, especially in the case of very fine sampling.

In the case of a spectral estimate, one writes

$$
\tilde{f}(x)=\sum_{k=-n / 2}^{n / 2} c_{k} e^{i 2 \pi k x / L}
$$

to be inserted into (5). As a result, a system of equations is obtained for the coefficients $c_{k} \in \mathbb{C}$. If $n=N$ and $\lambda=0$, the data are exactly interpolated (Fourier transformed), for $n \leq N$ and/or $\lambda \neq 0$, a spectral smoothing problem 
results. This can be solved (see App. A), but we will not consider this case. Instead of solving the complete smoothing problem in spectral space we follow a slightly different strategy: many experiments suggest that the noise sits predominantly in the high frequencies. Then a low-pass filter can be applied. A well-behaved standard filter is the Butterworth filter [29], which reads for the m-th order:

$$
B\left(k, k_{0}\right)=\frac{1}{1+\left(\frac{k}{k_{0}}\right)^{2 m}}
$$

where $k$ is the frequency, $k_{0}$ is the cutoff-frequency and $m$ defines the steepness of the filter. The two latter parameters must be related to the noise in the data. In the case of white noise, $k_{0}$ can be chosen just above the last $k$ which occurs from the dynamics of the system, the steepness determines how "fast" the noise amplitude is damped away spectrally after the cutoff frequency, this can be varied in each case, typical values lie between 6 and 10 [14]. So one simply performs a Fourier transformation, applies the spectral filter and transforms back. The derivative is then obtained in spectral space by multiplication with $(i 2 \pi k) / L$ (remember that $\left.y^{\prime}(x)=\sum_{k} \frac{i 2 \pi k}{L} c_{k} e^{i 2 \pi k x / L}\right)$. The complete representation of $\tilde{f}$ is

$$
\tilde{f}(x)=\sum_{k=-N / 2}^{k=N / 2} c_{k} B\left(k, k_{0}\right) e^{i 2 \pi k x / L}=\sum_{k=-N / 2}^{k=N / 2} \hat{c_{k}} e^{i 2 \pi k x / L}
$$

where $c_{k}$ are the coefficients obtained by Fourier transformation and $\hat{c_{k}}=$ $c_{k} B\left(k, k_{0}\right)$. It should be mentioned here that this representation also leads to a smoothing problem (5), cf. App. A. But instead of the computation of a complete set of coefficients $a_{j}$ one determines the cut-off frequency $k_{0}$ optimal for a given $\lambda$, since $k_{0}$ is the only parameter to be varied. This means that $\lambda$ and $k_{0}$ are equivalent and directly related as is shown in Appendix A. As an alternative to the Butterworth filter, one can use the Wiener filter, which is a good choice for linear processes. For scaling systems [30], wavelet transforms $[31,32]$ are a preferred choice. This underlines one obvious feature: the more information about the underlying process is known, the more details one uses for constructing the best method to estimate derivatives.

Both ways to solve Eq. (5), spline and spectral method, yield $n$ equations for $n$ unknowns ( $n$ being the number of knots for the smoothing splines or the number of basis function in the spectral method). The equations are overdetermined, since $N \geq n$ data points are available. This is resolved by the sum in the minimization procedure which eventually yields an $n \times n$ matrix. The procedure is similar to the usual linear regression $[14,26]$. If a Butterworth filter is applied, the matrices are reduced to dimension 1. 


\section{Numerical Results}

We compare the above methods by three examples, two using numerical data, one using experimental data: 1) the sine function, 2) the Lorenz system [33] in a chaotic state and 3) data from an acoustic measurement [16]. We quantify our results with the mean square error of the estimate of the first derivative and its smoothness (or equivalently its curvature). The dependence on the parameter of the methods are discussed in detail.

The variance

$$
\Delta=<\left(\tilde{f}^{\prime}\left(x_{i}\right)-f^{\prime}\left(x_{i}\right)\right)^{2}>
$$

is given as an overall measure for the results. The derivative is known exactly for the numerical data. As a second measure we use the curvature $s(f)$ from (4) or the difference

$$
S=(s(f)-s(\tilde{f}))^{2}=\left(\int_{a}^{b}\left|\tilde{f}^{\prime \prime}(x)\right|^{2} d x-\int_{a}^{b}\left|f^{\prime \prime}\left(x_{i}\right)\right|^{2} d x\right)^{2} .
$$

Other measures can be used, e.g., correlations or a norm different from $L_{2}$. We would like to point out that the characterization of results by (10) is common to local and global methods, but the latter use as additional constraint the smoothness. One of the main concerns of this publication is hint to the importance, from physical arguments and mathematical considerations, of this property.

For the finite differences we choose a second order method, where the parameter to be varied is the width $\delta x$. The approximation of the derivative reads

$$
\left(\frac{\partial \tilde{f}}{\partial x}\right)_{i}=\frac{4}{3} \frac{y_{i+l}-y_{i-l}}{2 \delta x}-\frac{1}{3} \frac{y_{i+2 l}-y_{i-2 l}}{4 \delta x}
$$

where $l$ is a positive integer determining the step width and $\delta x=l \Delta x$. The Savitzky-Golay filter was of fourth order; parameter dependence on the window size $n_{l}=n_{r}=n$ has been investigated. The spectral estimator has as parameters the cut-off $k_{0}$ and the number of basis functions, which is set here to $n=N$. The splines have the number of knots, $n$, used as parameter. The knots are, for the ease of use, space equidistant. Additionally, for splines and spectral method, one has the smoothing parameter to be varied. In the spectral case the variation of $\lambda$ is equivalent to the variation of $k_{0}$. According to the above, we approximate the function and then determine the derivative by one of the methods under consideration.

A comparison of the used methods requires a scaling of the parameters. For the finite difference method we use the quantity $w_{F D}=4 l \Delta x=4 \delta x$ the distance between the leftmost and the rightmost point in the considered domain. For Savitzky-Golay filter we choose the window size $w_{S G}=(2 n+1) \Delta x$. $w_{S G}$ 


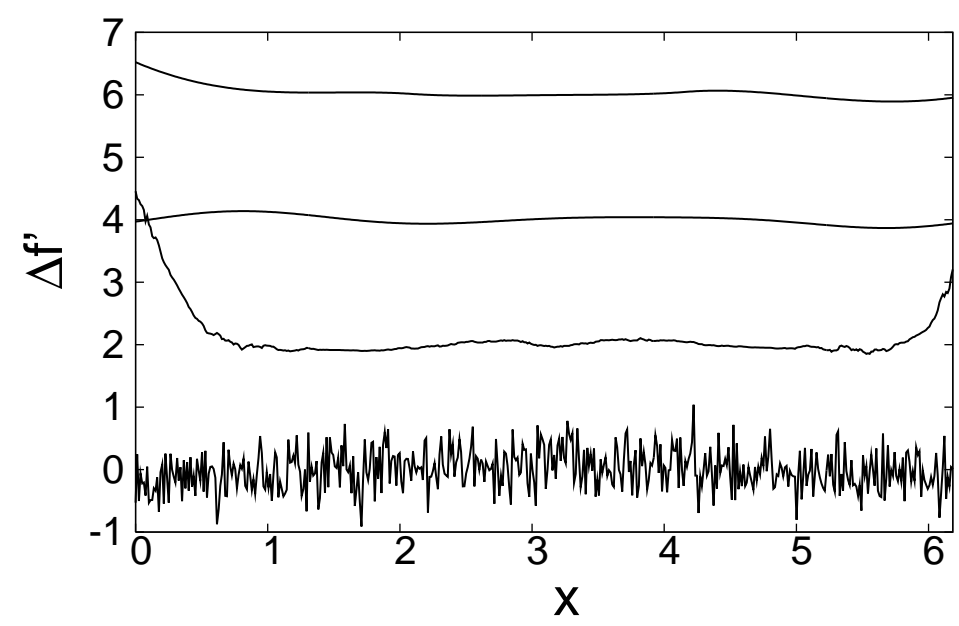

Fig. 1. Difference $\Delta f^{\prime}\left(x_{i}\right)=\tilde{f}^{\prime}\left(x_{i}\right)-f^{\prime}\left(x_{i}\right)$ of the estimate and the exact derivative $f^{\prime}(x)=\cos (x)$. Measurement noise was simulated by a Gaussian white noise process with standard deviation $\sigma=0.5$. From bottom to top: 1 . second order finite difference method $\left(\delta x=1.55, w_{F D}=6.18\right) 2$. Savitzky-Golay-filter $(n=143$, $\left.w_{S G}=3.61\right), 3$. spectral method $\left(k_{0}=2.99, w_{S}=2.09\right)$ and 4. smoothing splines $\left(n=7, \lambda=0.13, w_{S M}=0.89\right)$. Parameters for the methods were chosen such that they were optimal in the sense of Eq. (10). The offsets 0, 2, 4, 6 are added respectively for better visibility.

which corresponds directly to $w_{F D}$. For the spectral method, we define the window size by the wavelength of the cut-off, $w_{S}=L / k_{0}($ since $n=N)$. For the smoothing splines, finally, the typical window can be described by $w_{S M}=L / n$, the distance between two knots.

\subsection{Sine Function}

As a first example we use the function $f(x)=\sin (x)$ defined on the interval $0 \leq x \leq 2 \pi$ with added Gaussian white noise $\eta_{i}$ with standard deviation $\sigma$ and zero-mean. The data set consists of 500 Points $y_{i}=f\left(x_{i}\right)+\eta_{i}$, so that $\Delta x=2 \pi / 500$. In Fig. 1 the result for the derivative estimate is displayed $(\sigma=0.5)$. Finite differences $\left(\delta x=1.55, w_{F D}=6.18\right)$ yield an unacceptable result with extreme fluctuations of the order of 0.1 . Also, the result for the Savitzky-Golay filter $\left(n=143, w_{S G}=3.61\right)$ is not smooth and deviates heavily at the boundaries. Spectral $\left(k_{0}=2.99, w_{S}=2.09\right)$ and spline $(n=7, \lambda=0.13$, $\left.w_{S M}=0.89\right)$ methods, apparently work better.

Up to now, we showed the result for a specific set of parameters for each method. For a complete analysis, the dependence of the different methods on their parameters needs to be investigated. We varied the parameters over a wide range and considered the mean square error (10), cf. Fig. 2. For every 


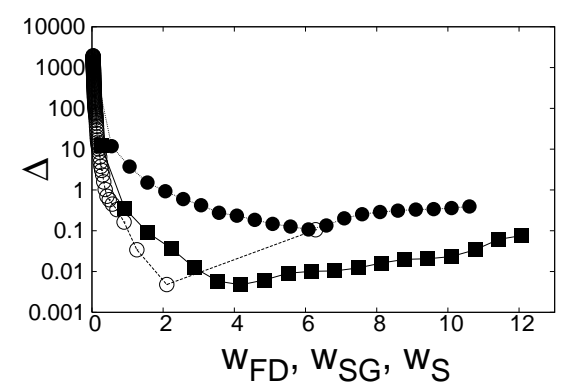

(a)

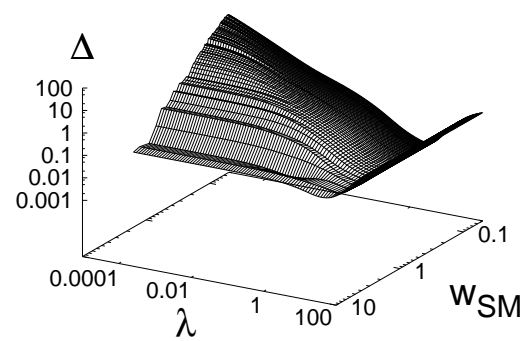

(b)

Fig. 2. Parameter dependence of the mean square error of the derivative of $\sin (x)$ with additive with noise $\sigma=0.5$. (a) For the finite differences, Savitzky-Golay-filter and the spectral method. - - finite difference method, the according parameter is $w_{F D}, \mathbf{\square}$ - Savitzky-Golay-filter $\left(w_{S G}\right)$, o - spectral method $\left(w_{S}\right)$. (b) mean square error for the smoothing splines in dependency of $w_{S M}$ and $\lambda$.

method a minimum occurs at the point of optimal approximation in the leastsquares sense.

For the finite difference method we varied the width $\delta x$. The optimal width is $\delta x=1.55\left(w_{F D}=6.18\right)$, nearly $1 / 3$ of the domain. Smaller spacing results in larger fluctuations, whereas an increasing spacing will not approximate the desired derivative. It is clearly visible from Fig. 1 that finite differences show the strongest fluctuations among all considered techniques. In practice finite differences should not be the first choice.

For the Savitzky-Golay-filter we varied the window size to find an optimum at $n \approx 143\left(w_{S G}=3.61\right)$. For a larger window the filter smoothes the function too much, and the result tends to a constant. For a smaller window the influence of noise becomes locally more important.

For spectral differentiation, the filter cut-off $k_{0}$ can be varied from 0-250 to determine the optimal cut-off. We find $k_{0} \approx 3\left(w_{S}=2.09\right)$. The original function, $\sin (x)$ implies that only $k=1$ is active in spectral space; for a top-hat filter with sharp edges this would result in $k_{0}=1$. Because $B\left(k, k_{0}\right)$ is smooth, a slightly larger cut-off is found. For higher cut-off values the approximation increasingly oscillates around the optimal solution. For spectral differentiation, in general, problems near boundaries occur, if the data are not perfectly periodic. In this case some data points close to the boundaries should be discarded after the determination of the derivative, but it might be better to switch to splines or other basis functions.

For smoothing splines, the mean square error depends on the number of used knots and the smoothing parameter. The optimal values are $\lambda \approx 0.13$ and $k=7\left(w_{S M}=0.89\right)$. When the smoothing parameter is increased the estimate consistently tends to a constant, when it is decreased the estimate represents a bigger part of the noisy fluctuations. The meaning of the number of used 


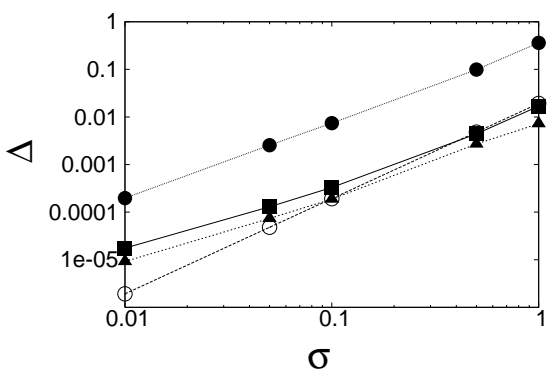

(a)

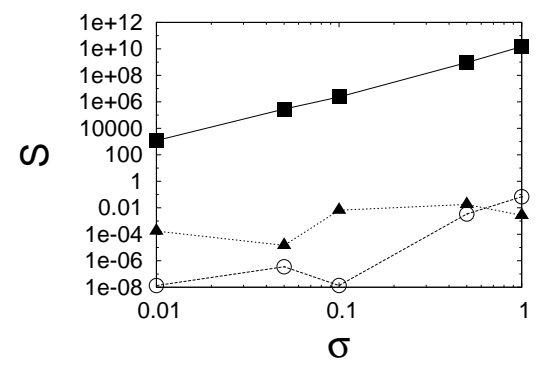

(b)

Fig. 3. Characterization of the derivative estimate of the sine data for different noise levels. a) least squares error, b) curvature $S$. On the x-axis the noise level of the white Gaussian noise added to the signal is plotted. $\bullet$ - finite difference method, - Savitzky-Golay-filter, $\boldsymbol{\Delta}$ - smoothing splines, o - spectral method.

knots for the smoothing splines can be understood as the degree of freedom of the smoother; if there is, e.g., one oscillation in the data, one needs minimal 3 knots for approximation. More oscillations require more knots, corresponding to a higher resolution, e.g., 10 oscillations can not be resolved with a smoothing spline of 10 knots; for details see [12]. In this sense the degree of freedom of the smoother can be understood as ability to fit a given number of oscillations in the data. If the number of knots exceeds the number of oscillations, one is faced with the bias-variance problem that all methods have in common.

For a direct comparison of the investigated methods for numerical differentiation we studied the dependence of the mean square error (10) on the noise level $\sigma$ for each method. Results are shown in Fig. 3a. Finite differences have an error about one order of magnitude larger than the other methods. The spectral method works very well for a small noise level, with an error of one order smaller than the Savitzky-Golay-filter and smoothing splines. For a higher noise contamination, however, smoothing splines are the best choice, whereas spectral methods and the Savitzky-Golay-filter are comparable by means of (10). Nevertheless, from a mathematical point of view, the Savitzky-Golay method is a local approximation, with no smooth relation between $\tilde{y}^{\prime}\left(x_{i}\right)$ and $\tilde{y}^{\prime}\left(x_{i+1}\right)$. This is also visible in Fig. 1 where at several points jumps can be observed.

To quantify the intuition on smoothness from the graphs (Fig. 1), we studied the dependence of the curvature $S$ on the standard deviation of the noise, cf. Eq. (11). The estimate was interpolated by a spline and then $S(\tilde{f})$ has been computed from (4) by averaging $\left|\tilde{f}^{\prime \prime}(x)\right|^{2}$ over each interpolated data point. The interpolation is necessary to determine the second derivative $\tilde{f}^{\prime \prime}$. One obtains $s(f)$ analytically, so that $S$ is easily determined. The results are shown in Fig. 3b. The curvature of the Savitzky-Golay-filter is some orders of magnitude worse than the one for the spectral method and the smoothing splines. Finite difference techniques are worse than Savitzky-Golay-filter, so 


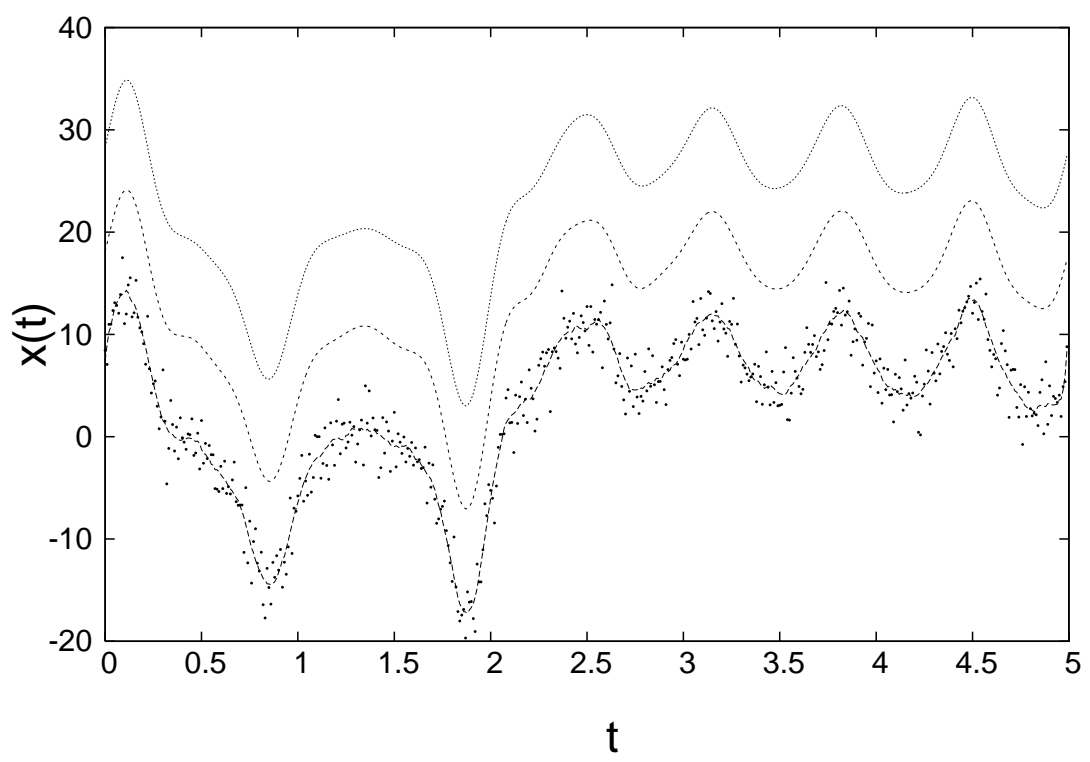

Fig. 4. Time dependency of the $x$-component of the Lorenz system, with additive with noise $\sigma=2.0$. Also shown are the results of the regression: Savitzky-Golay filter with $w_{S G}=0.47$ (bottom), spectral method $w_{S}=0.25$ (middle) and smoothing splines $w_{S M}=0.13$ (top). An offset is added for better visibility.

that we did not consider it here. The main difference between the methods is the different behavior of the smoothness, or the curvature, respectively. While for the Savitzky-Golay-filter one can clearly see a linear relation between noise and smoothness, for the spectral method and the splines the relation is roughly a constant, up to a certain, parameter-dependent noise level. One recognizes large fluctuations of $S$, resulting from over-smoothing the fit.

\subsection{Lorenz System}

As a second example we analyzed the $x$-component of the Lorenz system (Fig. 4),

$$
\begin{aligned}
& \dot{x}=\sigma(y-x) \\
& \dot{y}=R x-y-x z \\
& \dot{z}=-b z+x y .
\end{aligned}
$$

We integrate the system numerically by a Runge-Kutta algorithm of fourth order with time-step 0.01 and parameters $\sigma=10, R=28, b=8 / 3$. The integration was performed over 500 steps only. We also added Gaussian white noise to the data. The $x$ component of the trajectory of (13) is shown in Fig. 4. Results for the dependence of the mean square error on the level of noise are

similar to the previous case, $f(x)=\sin (x)$ (see Fig. 6). For small noise the 


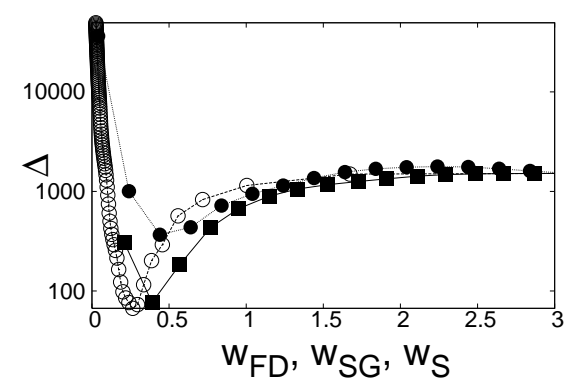

(a)

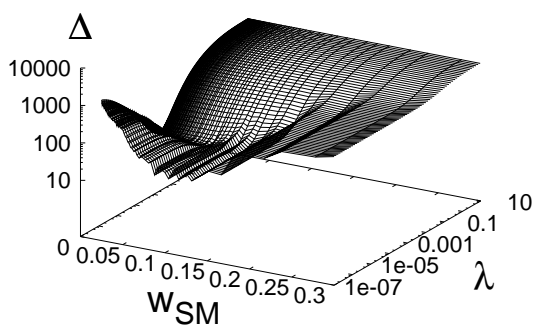

(b)

Fig. 5. Parameter dependence of the least squares error for the $x$-component of the Lorenz system with additive white noise $\sigma=2.0$ (a) For the finite differences, Savitzky-Golay-filter and the spectral method. - - finite difference method, the according parameter is $w_{F D}, \mathbf{\square}$ - Savitzky-Golay-filter $\left(w_{S G}\right)$, o - spectral method $\left(w_{S}\right)$. (b) least squares error for the smoothing splines in dependence on the number of knots $k$ and the smoothing parameter $\lambda$.

spectral method provides the smallest approximation error. Finite differences are here of the same order of magnitude.

Because in a chaotic time series, many scales are present with a broad spectrum, the Lorenz system is a good candidate for a study of the parameter dependence of the estimates under the aspect of a scaling system. We show the comparison of the methods in Fig. 5. The optimal parameters found here are different from the values for the sine. This is explained by the different number of oscillations. The data set for $\sin (x)$ contains exactly one oscillation, whereas the data for the Lorenz system contains 6 oscillations. As mentioned above the parameters must be chosen such that the bias-variance trade-off encountered.

For the Savitzky-Golay-filter, $w_{S G}=3.61$ for $\sin (x)$, whereas $w_{S G}=0.47$ for the Lorenz system. This difference can be explained by the fact that large window sizes will use many points for fitting a polynomial of 4 th order to each data point. For data sets with many oscillations this will result in bad approximation of the derivative or the function. For the smoothing splines $w_{S M}=0.89$ for $\sin (x)$, whereas for the Lorenz system $w_{S M}=0.13$. To resolve many oscillations, obviously more knots are needed. In the spectral case the dependency of the cut-off is $w_{S}=2.09$ for $\sin (x)$ and $w_{S}=0.26$ for the Lorenz system. Summarizing this subsection, the results for the much more complicated chaotic time signal match the ones for the quite simple sine signal. This can be interpreted as a sign for the generality of the results.

For a comparison of numerical differentiation methods with different data series one usually rescales the $x$ axis (in case of the Lorenz system the time $t$ ) by $\tilde{x}=x N_{\text {peak }} / L$, where $N_{\text {peak }}$ is the number of peaks. Then, the regression parameters $w_{F D}, w_{S G}, w_{S M}$ and $w_{S}$ are of the same order. But furthermore it should be noted, that every data series possesses characteristic edges, which 


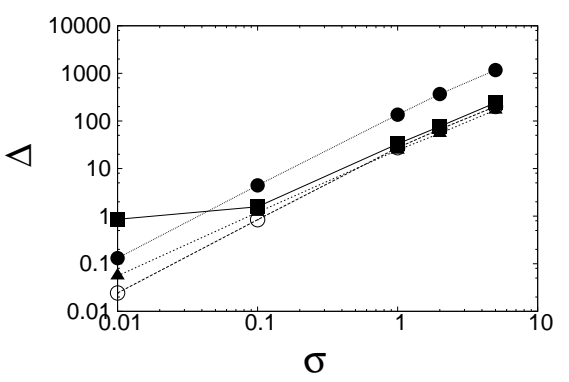

(a)

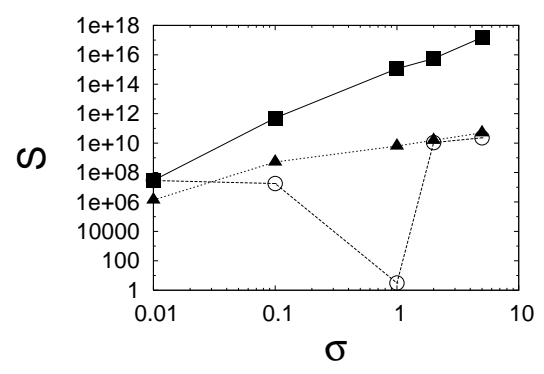

(b)

Fig. 6. Dependence of the least squares error and the smoothness on the additive noise standard deviation for the $x$-component of the Lorenz system, Eq. (13). a) least squares error, b) curvature $S$. On the x-axis the noise level of the white Gaussian noise added to the signal is plotted. $\mathbf{0}$ - Savitzky-Golay-filter, $\boldsymbol{\Delta}$ - smoothing splines, o - spectral method. The parameters for the fit are chosen to be optimal, cf. Fig. 5.

will naturally result in different parameters. If the time series also consists of intermittent domains, the situation is even worse. Then $N_{\text {peak }} / L$ is not a measure for the mean 'wavelength' of the data, and the domains, containing the oscillations would be over-smoothed. A detailed discussion of such phenomena goes far beyond the scope of this article.

\subsection{Experimental Data}

As the last example we analyzed experimental data from the acoustical signal emitted from the mouth of an organ pipe. This measurement is needed, if the complex acoustical system of an organ pipe shall be modeled as a nonlinear oscillator [34] to be found numerically by nonparametric data analysis $[35,36]$. Basically, the physics of the measured data it is not very important for our purposes and we will not comment further on the origin of our data, details are found elsewhere [16]. The time series $y(t)$ consists again of 500 points, the sampling interval is $\Delta t=1 / 44100 \mathrm{~s}$. In contrast to the previous examples, we do not have separate access to the derivatives and the measures (10) and (11) can not be applied to determined the regression parameters. So, one needs to estimate the optimal parameters. In principle, there are methods like generalized cross-validation dealing with this problem [26,28,37,38]. We will now explain briefly the functioning of generalized cross-validation (GCV) to have a complete presentation, an explicit application to our data lies beyond the scope of this article and is subject to future work.

GCV works by dropping one point $\left(y_{i}, x_{i}\right)$ from the data (in general $M$ points), the estimate is then based on the remaining $N-1$ points. The GCV is con- 


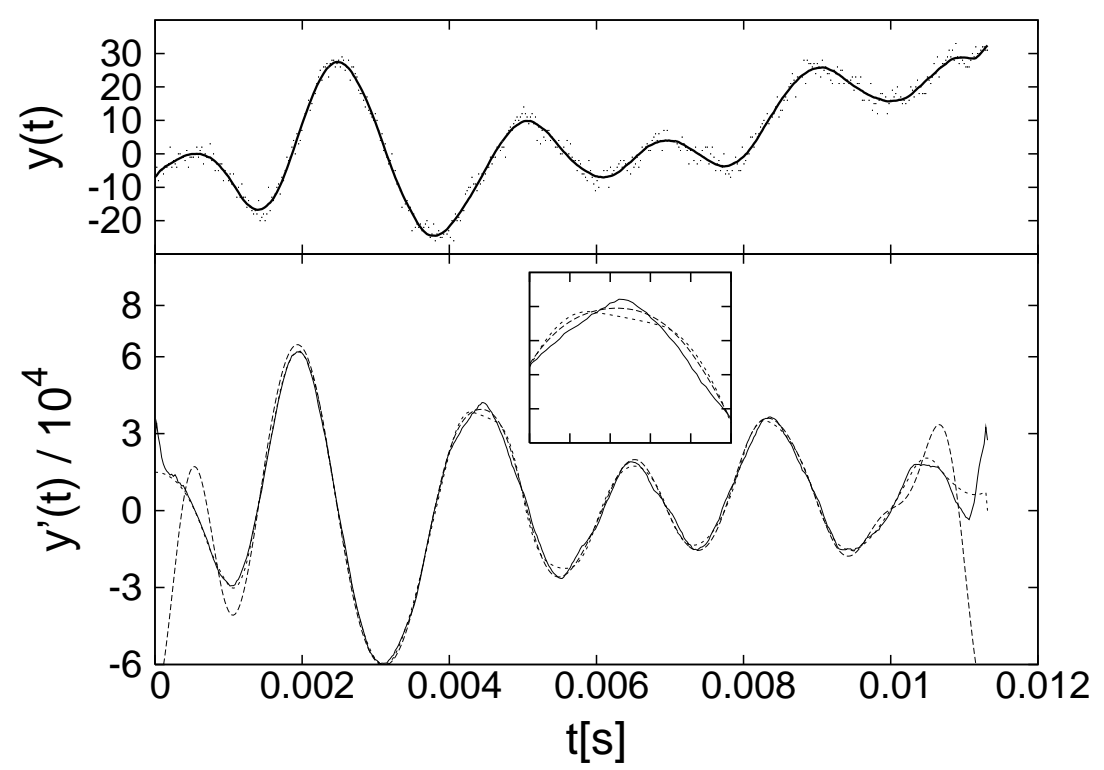

Fig. 7. Upper plot: data points of the organ pipe measurements and fitted function using Savitzky-Golay filter. The other methods are indistinguishable by eye. Lower plot: fitted derivatives. Solid line: Savitzky-Golay filter, dashed line: spectral filtering, dotted line: smoothing splines. The inset shows a part of the fitted derivative in the interval $0.04 s \leq t \leq 0.05 \mathrm{~s}$ Without quantitative analysis it is hard to say which curve is best, differences are however recognizable. The parameter used for fitting are $w_{S G}=1.6 \cdot 10^{-3} s$ for Savitzky-Golay filter, $w_{S}=8.7 \cdot 10^{-4} s$ for the spectral method and $w_{S M}=3.2 \cdot 10^{-4}$ and $\lambda=2.6 \cdot 10^{-11}$ for the smoothing splines.

structed by sum of squares

$$
G C V(\lambda)=\frac{1}{N} \sum_{i=1}^{N}\left\{y_{i}-\tilde{f}_{\lambda}^{-i}\left(x_{i}\right)\right\}^{2} .
$$

$\tilde{f}_{\lambda}^{-i}$ is the estimate for $f(x)$ if the point $\left(y_{i}, x_{i}\right)$ is omitted. The optimal parameter is calculated from $G C V$ for a number of values of $\lambda$ over a suitable range, then the minimizing $\lambda$ is selected. In other words, Eq. (14) gives an empirical estimate of the combined systematic errors, induced by the choice of the parameters, and statistical errors due to noise in the data. The parameter is chosen to minimize that estimate. Note that this is done for the estimated function, not the derivative.

Results for the estimates of function and derivative of the experimental data are shown in Fig. 7. For the experimental data we are faced with the problem that we do not know the true derivative. So, we have to compare the estimation, or construction of the function itself in terms of least-squares error $e=<(\tilde{f}(x)-f(x))^{2}>$ and the corresponding curvature $s$. All methods approximate the function quite well in terms of the least-squares criterion. As well the derivative seems well estimated, but slight differences are recognized. First, one notices the weakness of the spectral estimator at the boundaries; 
as well splines and Savitzky-Golay filter should be considered with great care in this region, as we already saw in the previous sections. By eye, all three estimates appear indistinguishable. However, if we estimate the curvature we find great differences.

We have two competing quantities in our considerations, the least-squares error, and the curvature. To compare either of them, one should fix the other one, correspondingly we need to fix the least-squares error to obtain quantitative statements about $s$. cf. Eq. (4). We -quite arbitrarily- chose the value $e=2$. Then, we searched in the whole parameter space of all the methods for fits with the least-squares error close to 2 and compared the according curvature. The Savitzky-Golay technique yields $e=2.04$ and the corresponding curvature $s=2.05 \cdot 10^{15}$, the window size $w_{S G}=1.61 \cdot 10^{-3}$. For the spectral estimator, we obtained $e=2.03$ with a curvature of $s=6.16 \cdot 10^{13}$ at $w_{S}=8,76 \cdot 10^{-4}$. The spline estimator yeld an error $e=2.05$ with $s=5.52 \cdot 10^{13}$ and $w_{S M}=3.23 \cdot 10^{-4}$. As a result we find again that the global methods are smoother by two orders of magnitude in comparison with the Savitzky-Golay method. Obviously, as far as a smooth curve is concerned the global smoothers are superior to the local methods under consideration. If smoothness is not relevant, all three methods are equivalent if the boundaries are neglected.

\section{Conclusion}

We presented a qualitative and quantitative comparison of local and global methods for the numerical estimation of derivatives. Whereas local methods are appealing due to their simplicity and easy implementation, we advocate for global methods, because the properties of the functions can be defined in a proper way. We focused on the important constraint of smoothness (expressed by the curvature) and showed how a corresponding minimization problem is solved. Furthermore, we demonstrated that global methods are superior to local ones if high-precision estimates are needed or measurement noise is large. We did not want to consider in detail the computational cost. But it shall be mentioned that a global estimate can be expensive if the number of points exceeds $10^{5}$. Then, programming skill (or large memory) is required to encounter the problem of large matrices to be multiplied.

We compared in this article finite differences, Savitzky-Golay filtering, smoothing splines and smoothing spectral estimators. To compare the methods, the dependence on parameters has been investigated, optimal parameters could be determined. The dependence on additive noise has been studied in detail and we found enormous differences in the methods. One result is that finite differences are orders of magnitudes off in comparison to the other methods. It is used as a worst case demonstration in this article. In terms of the least- 
squares error the remaining three methods are comparable in the sense that they are of the same order of magnitude. However in terms of smoothness, the Savitzky-Golay filter fails by some orders of magnitude, and only the global methods work well. It is remarkable that we can compare the methods on a logarithmic scale, i.e., techniques differ really to a huge extent. A more detailed look shows that spectral estimators work very well for small noise levels. For high noise levels, smoothing splines yield better results for the investigated systems.

Under a more general perspective, we showed how one can choose among some set of basis functions for the representation of the estimate. Obviously, for periodic functions a spectral representation is natural, similarly if one is interested in higher derivatives. If other boundary conditions are required, other basis functions might be favorable. Smoothing splines are an optimal choice if twice differentiability (or smoothness) is required, and no further information is available. For all basis systems the general procedure described above applies, formulated as a minimization problem. In principle one can imagine further constraints like minimal variance of the first derivative or other criteria. Those conditions can be easily built into the method as additional Lagrange multiplier.

From a practical point of view one has to decide how important it is to obtain smooth functions to a reasonable accuracy. For a rough guess, a local filter might do, for any high-precision analysis the implementation of the minimization, or smoothing problem does pay off. E.g., if one wants to process further the obtained derivatives, small differences can yield enormous changes in the final results. Our interest started with an application in some reconstruction techniques [39], where local methods are by far too inexact. Similar holds for prediction problems where the integration of functions based on the estimate of the derivatives is important [40].

\section{Acknowledgments}

We thank M. Rosenblum and M. Hanke-Bourgeois for helpful discussion. M. Abel and K. Ahnert acknowledge support by the DFG (German research foundation) (Proj. Nr. AB143/3). We are very grateful to S. Bergweiler for providing the experimental data. 


\section{A Appendix: Spectral smoother}

In the following, we assume that $x \in[0,1]$ for the measured data $\left(x_{n}, y_{n}\right) ; n \in$ $1, \ldots, N$

The spectral representation of a function reads

$$
f(x)=\sum_{k=0}^{N-1} c_{k} e^{i 2 \pi k x} .
$$

and the smoothing term in Eq. (5) reads

$$
\begin{aligned}
\lambda \int_{0}^{1}\left|f^{\prime \prime}(x)\right|^{2} d x & =\lambda \int_{0}^{1} f^{\prime \prime}(x) f^{\prime \prime}(x)^{*} d x \\
& =16 \lambda \pi^{4} \sum_{k, l=0}^{N-1} c_{k} c_{l}^{*} k^{2} l^{2} \int_{0}^{1} e^{i 2 \pi(k-l) x} d x \\
& =16 \lambda \pi^{4} \sum_{k=0}^{N-1} c_{k} c_{k}^{*} k^{4}
\end{aligned}
$$

where $\int_{0}^{1} e^{i 2 \pi(k-l) x} d x=\delta_{k l}$ is used. To solve the minimizing problem Eq. (5), we insert the above into

$$
\begin{aligned}
\chi^{2} & =\sum_{n=0}^{N}\left|y_{n}-f\left(x_{n}\right)\right|^{2}+\lambda \int_{0}^{1}\left|f^{\prime \prime}(x)\right|^{2} d x \stackrel{!}{=} \min . \\
& =\sum_{n=0}^{N}\left(y_{n}-\sum_{k=0}^{N-1} c_{k} e^{i 2 \pi k x_{n}}\right)\left(y_{n}-\sum_{k=0}^{N-1} c_{k}^{*} e^{-i 2 \pi k x_{n}}\right)+16 \lambda \pi^{4} \sum_{k=0}^{N-1} c_{k} c_{k}^{*} k^{4} .
\end{aligned}
$$

By variation of the coefficients we obtain the conditions

$$
\frac{\partial \chi^{2}}{\partial c_{k}}=0 \quad, \frac{\partial \chi^{2}}{\partial c_{k}^{*}}=0
$$

This yields the equations

$$
\begin{aligned}
& 0=-\sum_{n=1}^{N} e^{i 2 \pi k x_{n}}\left(y_{n}-\sum_{l=0}^{N-1} c_{l}^{*} e^{-i 2 \pi l x_{n}}\right)+16 \pi^{4} \lambda c_{k}^{*} k^{4} \\
& 0=-\sum_{n=1}^{N} e^{-i 2 \pi k x_{n}}\left(y_{n}-\sum_{l=0}^{N-1} c_{l} e^{i 2 \pi l x_{n}}\right)+16 \pi^{4} \lambda c_{k} k^{4}
\end{aligned}
$$

These are $2 \mathrm{~N}$ linear equations for the $2 \mathrm{~N}$ unknowns $\left\{c_{k}, c_{k}^{*}\right\}$ which can be solved by usual algebraic manipulation. 
In the case of using the Butterworth filter (8), one determines the Fourier coefficients $c_{k}$ in the conventional way [14]. Then the filter is applied. The only possible variation is in $k_{0}$ and a single equation results. For simplification we write $B\left(k, k_{0}\right)=B_{k}, \partial B\left(k, k_{0}\right) / \partial k_{0}=B_{k}^{\prime}$ and $c_{k} e^{i 2 \pi k x_{n}}=C_{k n}$ and obtain:

$$
\begin{aligned}
\frac{\partial \chi^{2}}{\partial k_{0}}= & 0 \\
= & -\sum_{n=1}^{N}\left(\sum_{k=0}^{N-1} B_{k}^{\prime} C_{k n}\left(y_{n}-\sum_{l=0}^{N-1} B_{l} C_{l n}^{*}\right)+\sum_{k=0}^{N-1} B_{k}^{\prime} C_{k n}^{*}\left(y_{n}-\sum_{l=0}^{N-1} B_{l} C_{l n}\right)\right)+ \\
& +32 \pi^{4} \lambda \sum_{k=0}^{N-1} B_{k} B_{k}^{\prime} k^{4} c_{k} c_{k}^{*} \\
= & -\sum_{n=1}^{N}\left(\sum_{k=0}^{N-1} B_{k}^{\prime} y_{n}\left(C_{k n}+C_{k n}^{*}\right)-\sum_{k, l=0}^{N-1} B_{k}^{\prime} B_{l}\left(C_{k n} C_{l n}^{*}+C_{k n}^{*} C_{l n}\right)\right) \\
& +32 \pi^{4} \lambda \sum_{k=0}^{N-1} B_{k} B_{k}^{\prime} k^{4} c_{k} c_{k}^{*}
\end{aligned}
$$

Using the definition of the Fourier components $c_{k}=\sum_{n=1}^{N} y_{n} e^{-i 2 \pi k x_{n}}$ this formula can be written as

$$
\frac{\partial \chi^{2}}{\partial k_{0}}=-2 N \sum_{k=0}^{N-1}\left(B_{k}^{\prime} c_{k} c_{k}^{*}+B_{k}^{\prime} B_{k} c_{k} c_{k}^{*}\right)+32 \pi^{4} \lambda \sum_{k=0}^{N-1} B_{k} B_{k}^{\prime} k^{4} c_{k} c_{k}^{*},
$$

where we use $1 / N \sum_{n=1}^{N} e^{i 2 \pi(k-l) x_{n}}=\delta_{k l}, 1 / N \sum_{k=0}^{N-1} e^{i 2 \pi k\left(x_{n}-x_{m}\right)}=\delta_{n m}$ and $\delta_{n m}$ the Kronecker delta. The factor $N$ can be avoided, if one scales $k_{0} \mapsto 2 \pi / N k_{0}$.

We write the minimum condition as $0=-F+\lambda G$. Then a simple relation $\lambda=F / G$ results, relating lambda to $k_{0}$. The inversion of this formula yields $k_{0}(\lambda)$. 


\section{References}

[1] H. Kantz and T. Schreiber. Nonlinear time series analysis. Cambridge University Press, 1997.

[2] M. D'Amico and G. Ferrigno. Comparison between the more recent techniques for smoothing and derivative assessment in biomechanics. Medical $\mathscr{E}$ Biological Engineering and Computing, 30:193-204, 1992.

[3] C. Schäfer, M.G. Rosenblum, H.-H. Abel, and J. Kurths. Synchronization in the human cardiorespiratory system. Physical Review E, 60:857-870, 1999.

[4] I. Z. Kiss, Q. Lv, and J. L Hudson. Synchronization of non-phase-coherent chaotic electrochemical oscillations. Physical Review E, 71(035201), 2005.

[5] M. Abel, S. Bergweiler, and K. Ahnert. Reconstruction of synchronizing time series in acoustical systems. Phys. Rev. Lett., 2007. in preparation.

[6] Markus Raffel, Chris Willert, and Jürgen Kompenhans. Particle Image Velocimetry. Springer Series on Experimental Fluid Mechanics. Springer, Berlin, 2001 ( 2nd edition).

[7] G. Conway. priv. comm.

[8] Jane Cullum. Numerical differentiation and regularization. SIAM Journal on Numerical Analysis, 8(2):254-265, 1971.

[9] R. S. Anderssen and P. Bloomfield. Numerical differentiation procedures for non-exact data. Numerical mathematics, 22:157-182, 1974.

[10] Robert Anderssen and Markus Hegland. For numerical differentiation, dimensionality can be a blessing! Mathematics of Computation, 68(227):11211141, 1999.

[11] M. Hanke and O. Scherzer. Inverse problems light: Numerical differentiation. American Mathematics Monthly, 108:512-521, 2001.

[12] G. Wahba. Spline Models for Observational Data. CBMS-NSF Regional Conference Series in Applied Mathematics 59. SIAM, Philadelphia, 1990.

[13] C.W. Groetsch. The Theory of Tikhonov Regularization for Fredholm Equations of First Kind. Pitman, London, 1984.

[14] W. H. Press, S. A. Teukolsky, W. T. Vetterling, and B. P. Flannery. Numerical Recipes in C: The Art of Scientific Computing. Cambridge University Press, Cambridge, 2nd edition, 1993.

[15] L. N. Trefethen. Finite Difference and Spectral Methods for Ordinary and Partial Differntial Equations. unpublished text, available at http://web.comlab.ox.ac.uk/oucl/work/nick.trefethen/pdetext.html, 1996. 
[16] M. Abel, S. Bergweiler, and R. Gerhard-Multhaupt. Synchronization of organ pipes by means of air flow coupling: experimental observations and modeling. J. Acoust. Soc. Am., 119(4), 2006.

[17] A. Torcini, H. Frauenkron, and P. Grassberger. A novel integration scheme for partial differential equations: an application to the complex Ginzburg-Landau equation. Physica D, 103:605, 1997.

[18] D. Goldberg. What every computer scientist should know about floating point arithmetic. ACM Computing Surveys (CSUR), 23(1):5-48, 1991.

[19] J. Stoer and R. Burlisch. Introduction to Numerical Analysis. Springer-Verlag, New York, 2nd edition, 1993.

[20] T. Sauer, J. A. Yorke, and Martin Casdagli. Embedology. Journal of Statistical Physics, 65(3/4):579-615, 1991.

[21] T. Wei, Y. C. Hon, and Y. B. Wang. Reconstruction of numerical derivatives from scattered noisy data. Inverse Problems, 21:657-672, 2005.

[22] Härdle W. Applied Nonparametric Regression. Cambridge University Press, Cambrige, 1990.

[23] K. Levenberg. A method for the solution of certain problems in least squares. Quart. Appl. Math., 2:164-168, 1944.

[24] D. Marquardt. An algorithm for least-squares estimation of nonlinear parameters. SIAM J. Appl. Math., 11:431-441, 1963.

[25] J. H. Ferziger and M. Perić. Computational Methods for Fluid Dynamics. Springer, Berlin, 3rd edition, 2002.

[26] T.J. Hastie and R.J. Tibshirani. Generalized Additive Models. Chapman and Hall, London, 1990.

[27] J. Honerkamp. Stochastic dynamical systems. VCH, New York, 1994.

[28] C. Gu and G. Wahba. Minimizing GCV/GML scores with multiple smoothing parameters via the newton method. SIAM J. Sci. Statist. Comput., 12(2):383398, 1991.

[29] S. Smith. The Scientist and Engineer's Guide to Digital Signal Processing. California Technical Publishing, 1997.

[30] Th. Bohr, M. Jensen, G. Paladin, and A. Vulpiani. Dynamical Systems approach to turbulence. Cambridge Nonlinear Science Series. Cambridge Univ. Press, Cambridge UK, 1998.

[31] M. Holschneider. Wavelets: An Analysis Tool. Oxford Mathematical Monographs. Oxford University Press, Oxford, 1995.

[32] A. Aldroubi and M. Unser. Wavelets in Medicine and Biology. CRC Press, Boca Raton, 1996. 
[33] E. N. Lorenz. Deterministic nonperiodic flow. Journal of Atmospheric Sciences, 20:130-141, 1963.

[34] B. Fabre and A. Hirschberg. Physical modeling of flue instruments: A review of lumped models. Acustica - Acta Acustica, 86:599-610, 2000.

[35] M. Buenner H. Voss and M. Abel. Identification of continuous spatiotemporal systems. Phys. Rev. E, 57(3), 1998.

[36] M. Abel. Nonparametric modeling and spatiotemporal dynamical systems. Int. J. Bif. Chaos, 14(6), 2004.

[37] A. C. Davison. Statistical models. Cambridge series in statistical and probabilistic mathematics. Cambridge Univ. Press, Cambridge UK, 2003.

[38] P. J. Green and B. W. Silverman. Nonparametric Regression and Generalized Linear Models. Chapman and Hall, London, 1994.

[39] M. Abel, K. Ahnert, J. Kurths, and S. Mandelj. Additive nonparametric reconstruction of dynamical systems from time series. Physical Review E, 71(015203), 2005.

[40] H. D. I. Abarbanel, M. E. Gilpin, and M. Rotenberg. Analysis Of Observed Chaotic Data. Springer, New York, 1997. 